Guaraglia, D. y Ravier, A. (2018). “'SE JUSTIFICA EL DESARROLLO DE UN ÍNDICE DE PRECIOS EN LA PAMPA?" Revista Perspectivas de las Ciencias Económicas y Jurídicas. Vol. 8, N 1 (enerojunio). Santa Rosa: FCEyJ (UNLPam); EdUNLPam; ISSN 2250-4087, e-ISSN 2445-8566 pp. 27-41. DOI http://dx.doi.org/10.19137/perspectivas-2018-v8n1a02

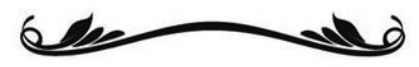

¿SE JUSTIFICA EL DESARROLLO DE UN ÍNDICE DE PRECIOS EN LA PAMPA?

IS THE DEVELOPMENT OF A PRICE INDEX JUSTIFIED IN THE PAMPA?

Por Darío GUARAGLIA

y Adrián RAVIER²

\title{
Resumen
}

Analizamos las ventajas y desventajas de desarrollar un índice de precios local, provincial, tomando en cuenta la mayor transparencia que alcanzó el INDEC a partir de 2016. Concluimos que se justifica elaborar un índice para La Pampa dado que su realidad no se encuentra representada en el índice Nacional, y tampoco en los dos índices regionales Pampeano y Patagónico que se elaboran desde mediados de 2017. Se debe notar que el IPC de la región Pampeana tiene un fuerte sesgo bonaerense en un período histórico donde el gobierno nacional intenta corregir las bajas tarifas de Buenos Aires frente a las existentes en el interior del país. Nuevas correcciones en tarifas en Buenos Aires implican una mayor variación en los índices de precios Nacional y Región Pampeana de la que se presenta en la Provincia de La Pampa. Mostramos que el IPC de la Región Patagónica resulta más representativo de La Pampa que el IPC de la Región Pampeana.

Palabras clave: Cuentas nacionales, Indice de Precios al Consumidor, inflación

\begin{abstract}
We analyze the advantages and disadvantages of developing a local, provincial price index, taking into account the greater transparency reached by INDEC as of 2016 . We conclude that an index for La Pampa is justified given that its reality is not represented in the National index, and neither in the two Pampeano and Patagonian regional indexes that are prepared since mid-2017. It should be noted that the CPI of the Pampean region has a strong Buenos Aires bias in a historical period where the national government tries to correct the low rates of

\footnotetext{
${ }^{1}$ Universidad Nacional de La Pampa. Facultad de Ciencias Económicas y Jurídicas. Santa Rosa, La Pampa. Contador Público Nacional. dguaraglia@hotmail.com

2 Universidad Nacional de La Pampa. Facultad de Ciencias Económicas y Jurídicas. Santa Rosa, La Pampa. Docente. Lic. en Economía, Dr. en Economía Aplicada. adrianravier@yahoo.com.ar
} 
Buenos Aires compared to those existing in the interior of the country. New price corrections in Buenos Aires imply a greater variation in the National and Pampean Price indices of the one presented in the Province of La Pampa. We show that the CPI of the Patagonian Region is more representative of La Pampa than the CPI of the Pampean Region.

Keywords: National accounts, Consumer Price Index, inflation

Dada la intervención que sufrió el Instituto Nacional de Estadísticas y Censos (INDEC) durante el gobierno de Cristina Fernández de Kirchner, los indicadores generados por esta institución oficial perdieron credibilidad. A partir de entonces, numerosas provincias como San Luis o la Ciudad de Buenos Aires, la oposición en el Congreso de la Nación y consultoras privadas iniciaron el desarrollo de indicadores propios.

Un equipo de profesionales en la Facultad de Ciencias Económicas y Jurídicas de la Universidad Nacional de La Pampa (UNLPam) inició el proceso para elaborar su propio Indice de Precios al Consumidor en La Pampa (IPC-LP) en marzo de 2016, lo que sirvió para auditar el trabajo del INDEC y poder comparar los resultados con los índices disponibles. El trabajo contó con la asistencia técnica de profesionales del Instituto Interdisciplinario de Economía Política, dependiente de la Facultad de Ciencias Económicas de la Universidad Nacional de Buenos Aires y ofreció los primeros resultados en el último trimestre de 2016, pudiendo en 2017 conformar índices mensuales y también un resultado anualizado.

A partir de diciembre de 2015, y tras la asunción del nuevo gobierno nacional, se hizo manifiesta la intención por recuperar transparencia en la elaboración de indicadores, lo que se efectivizó en 2016 y 2017 notando resultados similares en los distintos indicadores que medían los precios.

En esta transición hacia una mayor confiabilidad en sus indicadores, el IPC de INDEC dejó de medir solamente Buenos Aires y pasó a ser un índice realmente nacional. A su vez, sustituyó la canasta con 9 divisiones, por otra con 12 divisiones, según las recomendaciones internacionales. A mediados de 2017, además, el INDEC comenzó a publicar índices regionales, incluyendo la región pampeana, la que comprende a la provincia de La Pampa, pero también a una parte de la Provincia de Buenos Aires y las provincias de Santa Fe, Entre Ríos y Córdoba.

Dada la escasez de recursos, y dado que se hizo efectiva la mayor transparencia en los indicadores de INDEC, resulta necesario evaluar la conveniencia de desarrollar un índice de precios local.

El trabajo se divide en tres partes. En la parte I ofrecemos una síntesis metodológica de cómo elaboramos el IPC-LP. En la parte II ofrecemos los resultados del IPC-LP y una comparación y análisis con otros indicadores semejantes. En la parte III ofrecemos las conclusiones.

\section{PARTE I:}

\section{Metodología del IPC-LP}

Los profesionales de la FCEyJ de la UNLPam que participamos en este proyecto nos 
regimos por principios éticos y metodológicos reconocidos y recomendados internacionalmente (véase Comisión Económica Europea, ECE/CES/2016/8, citado en INDEC 2016a):

1. INDEPENDENCIA PROFESIONAL: es decir la libre decisión sobre los procesos, fuentes y demás componentes de las operaciones estadísticas respecto de cualquier interferencia o presión de origen político.

2. IMPARCIALIDADY OBJETIVIDAD: las estadísticas oficiales deben ser desarrolladas, producidas y comunicadas de un modo neutral y confiable, libre de eventuales sesgos y todos los usuarios deben tener igual y simultáneo acceso a las mismas.

3. PRECISIÓNY CONFIABILIDAD: deben reflejar fielmente la realidad y basarse en criterios científicos para la selección de fuentes, métodos y procedimientos.

4. CLARIDAD Y TRANSPARENCIA: las estadísticas deben ser presentadas de un modo claro y comprensible. Los métodos y procedimientos aplicados deben ser transparentemente comunicados para facilitar una adecuada interpretación.

5. CONFIDENCIALIDAD ESTADÍSTICA: en las estadísticas los datos individuales que se refieran a individuos o personas jurídicas deben ser estrictamente confidenciales y usarse sólo con propósitos estadísticos.

\section{La canasta y su composición}

El primer paso en la elaboración del IPC-LP es definir los artículos que componen la canasta. Para ello hemos accedido a las Encuestas Nacionales de Gastos de los Hogares (ENGHO) que INDEC elaboró en 2004-05 y 2012-13, la que se construye a partir de cinco cuestionarios:

Cuestionario 1: Características de los Hogares

Cuestionario 2: Gastos diarios

Cuestionario 3: Gastos varios

Cuestionario 4: Gastos personales

Cuestionario 5: Ingresos

La ENGHO sintetiza en una serie de tablas o cuadros la información que surge de estos cuestionarios. Concretamente para este proyecto, los cuadros que para nosotros fueron relevantes sintetizan los artículos que los encuestados dicen consumir diariamente, junto con el monto que la población encuestada destinó a la compra de esos artículos. Esto permite definir la ponderación o peso que cada uno de estos artículos tiene en la canasta, que a su vez se compone de nueve divisiones:

1. Alimentos y bebidas

2. Indumentaria y calzado

3. Propiedades, combustibles, agua y electricidad

4. Equipamiento y mantenimiento del hogar

5. Salud

6. Transporte y comunicaciones 
7. Esparcimiento

8. Enseñanza

9. Bienes y servicios varios

A continuación ofrecemos un detalle descriptivo del contenido de cada una de estas divisiones, siguiendo las recomendaciones de INDEC (2016a):

- División 1. Alimentos y bebidas: incluye todos los alimentos y bebidas (alcohólicas y no alcohólicas) adquiridos para consumir dentro y fuera del hogar.

- División 2. Indumentaria y calzado: está conformado por indumentaria (abrigos, vestimenta interior y exterior para hombres, mujeres y niños), calzado (para hombres, mujeres y niños), accesorios y servicios (telas, hilados, artículos de mercería, reparación de vestimenta y calzado).

- División 3. Propiedades, combustibles, agua y electricidad: incluye el alquiler de la vivienda, gastos comunes y reparaciones, así como combustibles para usar en el hogar (gas envasado, gas de red, leña, entre otros), agua y electricidad.

- División 4. Equipamiento y mantenimiento del hogar: incluye los bienes para equipar el hogar (muebles, artefactos, electrodomésticos; vajilla, blanco y mantelería); los artículos para el mantenimiento del hogar (artículos de limpieza y herramientas) y los servicios para el mantenimiento del hogar (reparaciones de muebles, reparaciones de artefactos y electrodomésticos, tintorería, lavadero y servicio doméstico).

- División 5. Salud: incluye los productos medicinales y accesorios terapéuticos (medicamentos, elementos para primeros auxilios, aparatos y accesorios) y los servicios para la salud (sistema pre-pago de asistencia médica, consultas médicas y odontológicas, análisis clínicos y radiológicos).

- División 6. Transporte y comunicaciones: incluye la compra-venta de vehículos particulares, su funcionamiento y mantenimiento (combustibles, seguros, estacionamiento, etcétera), el transporte público, el correo y el teléfono.

- División 7. Esparcimiento: incluye los servicios de turismo, los equipos de audio, televisión, video y computación, los servicios de esparcimiento (espectáculos deportivos, cine, teatro, conciertos, cuotas del club deportivo, televisión por cable, etcétera), libros, diarios y revistas no profesionales; y otros bienes (equipos de cine y fotografía y sus accesorios, películas; juguetes y juegos, animales domésticos y artículos para deporte

- División 8. Enseñanza: incluye servicios educativos para la educación formal (cuota y aranceles para preescolar, enseñanza primaria, secundaria y universitaria) y no formal (idiomas, entre otros), así como textos y útiles escolares.

- División 9. Bienes y servicios varios: incluye cigarrillos, artículos de tocador, servicios para el cuidado personal, servicios financieros y otros bienes y servicios diversos. 


\section{Gráfico No. 1: Divisiones de la Canasta del IPC-LP con sus ponderaciones}

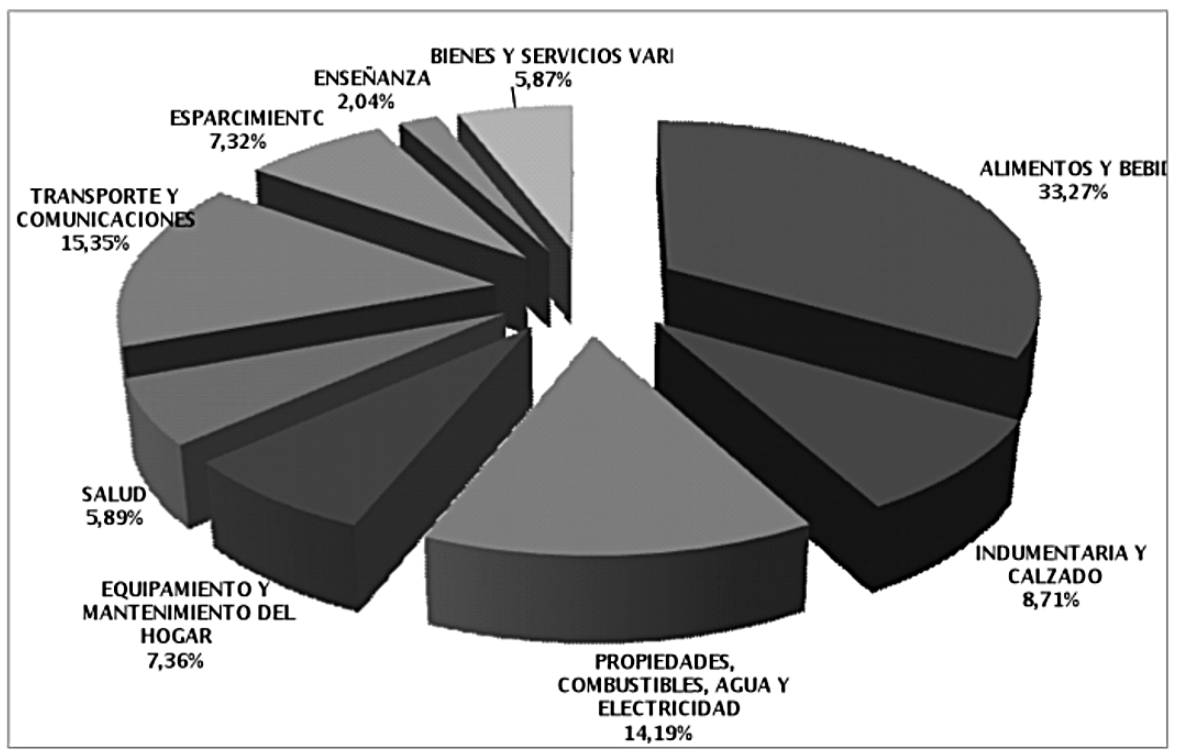

Fuente: Elaboración propia en base a microdatos de la ENGHO 2004-05 para La Pampa.

\section{Estructura de gastos en cinco niveles}

Cada división, a su vez, se desglosa en una estructura de gastos de cinco niveles, incluyendo división, grupo, clase, subclase y artículo.

Para hacernos de esta información utilizamos el sistema SPSS (versión 15) que nos permitió importar una base que sintetiza los micro-datos de la estructura de gastos mencionada, pero específicamente para los encuestados en la ciudad de Santa Rosa, La Pampa.

El cuadro No. 1 ofrece un extracto de esta estructura de gastos desagregada. De lo más de 1000 artículos que componen la canasta que resultó de la ENGHO 2004-05, nuestro equipo de trabajo seleccionó 320 artículos para que conformen la canasta sobre la cual se mide el IPC-LP. 


\section{Cuadro No. 1: Extracto de la Estructura de Gastos desagregada}

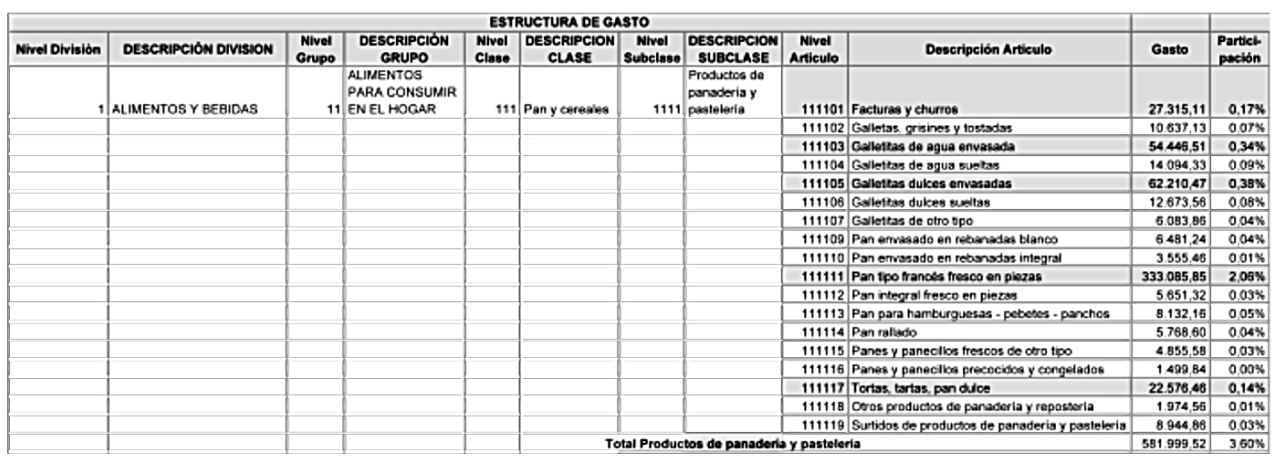

Fuente: Elaboración propia en base a microdatos de la ENGHO 2004-05 para La Pampa.

\section{Criterio para la selección de artículos}

El criterio para seleccionar estos artículos no fue arbitrario, sino que se basó en aquellos artículos que tienen mayor peso en la canasta según la estructura de gastos que se presentó en el cuadro anterior. Nótese en la última columna del cuadro anterior que los artículos marcados en el cuadro como "Facturas y churros", "Galletitas de agua envasada",

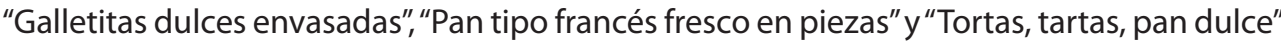
son precisamente los cinco artículos de mayor peso entre los que componen la sub-clase "Productos de panadería y pastelería".

La canasta de 320 artículos que conforma el IPC-LP representa más del $80 \%$ del gasto que surge de la encuesta en La Pampa, lo que es bastante representativo del consumo de los hogares local.

El porcentaje de los artículos excluidos se re-ponderó en aquellos que contaban con características más parecidos, siempre manteniendo intacto el porcentaje que arrojaron los micro-datos para la división, grupo, clase y sub-clase.

\section{Definición de atributos para cada uno de los 320 artículos}

El paso siguiente consistió en definir los atributos o las características específicas de cada uno de estos 320 artículos, como la marca y el gramaje a relevar. Como ejemplo, en el cuadro No. 2 tomamos los mismos cinco artículos del cuadro No. 1 y asignamos los atributos. 


\section{Cuadro No. 2: Atributos para 5 artículos seleccionados}

\begin{tabular}{|l|l|}
\hline Artículos & Atributos \\
\hline Facturas y churros & Medialunas de grasa o de manteca. Por docena \\
\hline Galletitas de agua envasada & Paquete de 3 unidades. Bagley, Traviata, 303 gramos. \\
\hline Galletitas dulces envasadas & Paquete de 1 unidad. Bagley, Sonrisas, 118 gramos. \\
\hline Pan tipo francés fresco en piezas" & Precio por kg (pan flauta) \\
\hline Tortas, tartas, pan dulce & Pasta frola de membrillo, 1000 gramos \\
\hline
\end{tabular}

Fuente: Elaboración propia.

\section{Lugar de compra}

Es importante también determinar el lugar de compra de estos artículos. Por ejemplo, las "facturas y churros" pueden comprarse en el supermercado, en una panadería o incluso en la vía pública, con diferencias de precios notables. Al efecto, la misma base de datos que se importó bajo el sistema SPSS nos ofreció también la ponderación sobre el lugar de compra de estos productos. Esto nos permite ponderar el peso relativo del precio que se releva en un supermercado frente al precio que se releva en un comercio minorista. En el cuadro No. 3 se puede observar un extracto del cuadro para los mismos cinco artículos de los cuadros anteriores:

\section{Cuadro. No. 3: Lugar de compra}

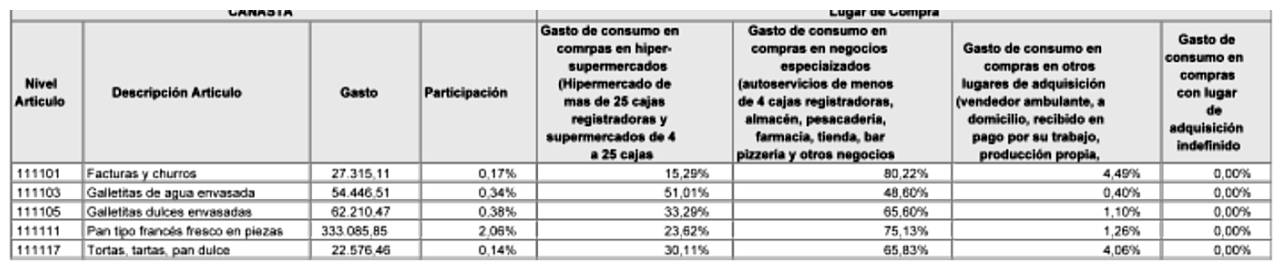

Fuente: Elaboración propia en base a microdatos de la ENGHO 2004-05 para La Pampa.

Nótese en la columna 7 que hay cierto gasto en Santa Rosa, La Pampa, en "otros lugares de adquisición" como vendedor ambulante, pero dada la dificultad de medirlo lo re-ponderamos entre el gasto de consumo en hiper y supermercados (caracterizado en contar con más de cuatro cajas) y gasto en consumo en negocios especializados.

Nuestro relevamiento, en definitiva, se concentró en supermercados y comercios minoristas, lo que nos obligó a contactar a 314 comerciantes, repartidos en la Ciudad de Santa Rosa y que mostraron muy buena predisposición para participar del proyecto, accediendo a ofrecer información sobre los precios que cobran por sus servicios y productos de forma mensual. 
Sin ánimo de ser exhaustivo, entre los informantes aparecen supermercados, autoservicios, panaderías, fábrica de pastas, carnicerías, avícolas, pescaderías, verdulerías, restaurantes, rotiserías, kioscos, heladerías, lencerías, lanería, comercios de venta de ropa de hombre, mujer y unisex, para niños, deportiva, billouteri, lavaderos, zapaterías, pinturerías, materiales para la construcción, mueblerías, mobiliarios de bebes, ferreterías, farmacias, ópticas, sanatorios y clínicas, concesionarios, neumáticos, empresas de ómnibus, encomiendas, correo postal, aseguradoras, bicicletería, viveros, alimento para mascotas, instrumentos musicales, canchas de fútbol, gimnasios, discotecas, librerías, fotocopiadoras, guardería, escuela primaria y secundaria privada, enseñanza artística, idioma extranjero, peluquería, artículos de higiene personal, pañaleras, salón de eventos, servicios profesionales, etcétera.

Desde luego habrá que añadir a esta lista el relevamiento a los cuadros tarifarios de los servicios públicos, que reviste un carácter fundamental en este período histórico de frecuentes correcciones tarifarias.

\section{Cuadro No. 4: Cantidad de informantes por división}

\begin{tabular}{|c|r|}
\hline DIVSION & CANIIDADDEINFORMANIES \\
\hline 1 & 57 \\
\hline 2 & 55 \\
\hline 3 & 21 \\
\hline 4 & 36 \\
\hline 5 & 21 \\
\hline 6 & 41 \\
\hline 7 & 32 \\
\hline 8 & 28 \\
\hline 9 & 23 \\
\hline TOTAL & 314 \\
\hline
\end{tabular}

Fuente: Elaboración propia

El cuadro No. 4 resume la cantidad de informantes que asignamos por división para cubrir los relevamientos que hacen posible el IPC-LP.

Se debe notar, que en algunos de estos comercios minoristas se releva sólo un dato o menos de una decena de datos, y en otros, como en los supermercados, se relevan cientos de datos. En conjunto, el IPC-LP releva unos 3000 precios por mes, lo que suma 36.000 precios en el año.

\section{ENGHO 2004-05 versus ENGHO 2012-13}

Respecto de la elección de la ENGHO 2004-05 frente a la ENGHO 2012-13, nuestra elección se ajustó a las recomendaciones de INDEC por la primera, considerando que la última 
no resultó confiable por el escaso nivel de respuestas en los cuestionarios (INDEC, 2016b). Se decidió trabajar con la ENGHO 2004-05, corrigiendo el sesgo que en aquel tiempo podían tener algunos artículos como el teléfono fijo y la telefonía móvil, y los servicios públicos. En el primer caso, se invirtió la baja ponderación que tenían los celulares por la alta ponderación que tenían los teléfonos fijos. En el segundo caso, se mantuvo el porcentaje de ponderación de la división 3, pero se re-ponderó internamente el peso de los servicios públicos en función del peso que alcanzaron estos servicios en la ENGHO 2012-13.

La siguiente referencia de un informe de INDEC respalda esta decisión metodológica:

“Durante el período de interés comprendido entre la realización de la ENGHo 2004/05 y el mes de diciembre de 2015 es posible encontrar una gran dispersión en los cambios de precios que se produjeron en distintos rubros, mientras que la actualización de las tarifas de servicios públicos se ha mantenido retrasada con respecto a otros rubros. Esta discrepancia en la evolución de los precios de los distintos rubros que conforman el índice generan cambios en los precios relativos que difícilmente podrían mantener invariables las estructuras de gastos durante más de 10 años. Sobre la base de esta discusión, la Dirección de Índices de Precios de Consumo ha decidido actualizar los ponderadores basados en la ENGHo 2004/05. La fundamentación de esta decisión se basa en recomendaciones y prácticas internacionales. Como el gasto $(\mathrm{g})$ se puede descomponer en un componente de cantidad (q) y otro de precio (p), y no se dispone de información actualizada sobre cantidades, la decisión de actualizar las ponderaciones lleva a que éstas se calculen a partir de valores de gastos híbridos, en los que las cantidades implícitas corresponden al período de referencia de la ENGHo y los precios corresponden al período base del IPC-GBA.

Para llevar a cabo el ajuste de las ponderaciones que surgen de la encuesta de gastos, se tomaron en cuenta los precios promedios implícitos en el cálculo del IPC-GBA base 1999 correspondientes a los meses de relevamiento de la ENGHo 2004/05. Estos precios promedio fueron comparados con precios promedio relevados durante diciembre de 2015. Para ello, se realizó un apareo de las variedades del IPC-GBA Base 1999 con respecto a las variedades del IPC-GBA actual, analizando de manera detallada las especificaciones de cada ítem de la canasta que correspondía a cada índice. De este modo, sólo se consideraron los relativos de precio correspondiente a los ítems cuyas especificaciones se consideraban similares. De otro modo, se hubieran reflejado cambios "impuros" en los precios promedio, indicativos de cambios en la calidad de los bienes y servicios considerados.

Finalmente, una vez actualizado el conjunto de ponderaciones para la canasta del nuevo índice, se realizaron algunos ajustes ad-hoc para reflejar de forma más adecuada los hábitos de consumo vigentes. Un ejemplo de este ajuste es el que corresponde al servicio telefónico. Según la ENGHo 
2004/05, los hogares destinaban mayor proporción de gasto a la telefonía fija que a la telefonía móvil. Hoy en día la situación es exactamente la contraria. En el mismo sentido, el gasto asociado al servicio de Internet domiciliario era menor en el año 2004/05. Para corregir estas distorsiones que podrían reflejar una estructura de gastos más antigua, se reponderó la participación de los servicios de telefonía fija, móvil e Internet domiciliaria. Dado que las empresas prestadoras de estos servicios son pocas y ofrecen todos los servicios, se utilizaron datos correspondientes a la facturación de las mismas asociadas a cada servicio para reasignar la ponderación del gasto agregado de servicio telefónico a cada uno de los servicios puntuales." (INDEC, 2016b, p. 20)

Como cierre de esta primera parte metodológica -y a modo de síntesis-, debemos resaltar que la canasta del IPC-LP cuenta con 320 artículos que se reparten en 9 divisiones y, a su vez, en grupos, clases y sub-clases. En promedio, se relevan y supervisan 150 precios por día (hábil), que hacen 3.000 al mes y 36.000 al año. Una vez los precios son cargados en un sistema, desarrollamos los cálculos correspondientes para llegar al resultado mensual, los que a su vez agregamos para llegar al resultado acumulado.

\section{PARTE II:}

\section{Resultados del IPC-LP y comparación con el IPC de INDEC}

El acumulado del IPC-LP desde enero a diciembre de 2017 ofrece un incremento del 23,9\%.

\section{Resultados mensuales del IPC-LP}

El cuadro No. 5 muestra los 12 resultados mensuales que arrojó el índice en 2017, confirmando un proceso de desinflación al comparar la media del primer semestre en 1,9\% con aquella del segundo semestre en 1,7\%.

\section{Cuadro No. 5: Resultados mensuales del IPC-LP - enero a diciembre de 2017}

\begin{tabular}{|c|c|c|c|c|c|c|}
\hline IPCLP 2017 & Ene & Feb & Mar & $\mathrm{Abr}$ & May & Jun \\
\hline & $2,0 \%$ & $2,2 \%$ & $2,4 \%$ & $1,9 \%$ & $1,5 \%$ & $1,4 \%$ \\
\hline & Jul & Ago & Sept & Oct & Nov & Dic \\
\hline & $15 \%$ & $122 \%$ & $1,9 \%$ & $1,4 \%$ & $1,7 \%$ & $2,5 \%$ \\
\hline
\end{tabular}

Fuente: IPC-LP, FCEyJ, UNLPam 


\section{Comparando el IPC-LP versus el IPC de INDEC Nacional y Regionales}

El cuadro No. 6 compara el IPC-LP con los índices de INDEC a nivel nacional, y también con dos de los datos regionales, a saber, la región pampeana y la Patagonia.

La región Pampeana comprende, además de La Pampa, una parte de Buenos Aires, Santa Fe, Entre Ríos y Córdoba.

EI IPC-LP ofreció, como ya dijimos, entre enero y diciembre un incremento del 23,9\% frente a un 24,8 \% de INDEC a nivel Nacional, 25,0\% de INDEC para la Región Pampeana y 23,5\% de INDEC para la región Patagónica. Es importante notar que el IPC-LP se encuentra $0,9 \%$ debajo del IPC Nacional, y a la vez, en medio de los dos IPC regionales.

\section{Cuadro No. 6: IPC-LP vs IPC de INDEC Nacional y Regionales}

\begin{tabular}{|c|c|c|c|c|}
\hline \multirow[t]{2}{*}{ IPCIP acumulado enero a diciembre de 2017} & \multirow[b]{2}{*}{$\begin{array}{c}\text { IPC } \\
\text { La Pampa }\end{array}$} & \multirow[b]{2}{*}{$\begin{array}{l}\text { IPCINDEC } \\
\text { Nacional }\end{array}$} & \multirow[b]{2}{*}{$\begin{array}{c}\text { IPCINDEC } \\
\text { R Pampeana }\end{array}$} & \multirow[b]{2}{*}{$\begin{array}{l}\text { PPCINDEC } \\
\text { Patagonia }\end{array}$} \\
\hline & & & & \\
\hline IFC & $23,9 \%$ & $24,8 \%$ & $25,0 \%$ & $23,5 \%$ \\
\hline División 1 -Alimentos y Bebidas & $18,7 \%$ & $20,4 \%$ y $23,7 \%$ & $19,1 \%$ y $23,7 \%$ & $16,9 \%$ y $22,5 \%$ \\
\hline División 2 -Indumentaria y Calzados & $9,8 \%$ & $16,6 \%$ & $19,1 \%$ & $14,6 \%$ \\
\hline División 3-Propiedades, Combustibles, Agua y Elect. & $41,4 \%$ & $55,6 \%$ & $56,4 \%$ & $48,8 \%$ \\
\hline División 4-Equipamiento y Mantenimiento del Hogar & $22,6 \%$ & $17,4 \%$ & $18,0 \%$ & $15,9 \%$ \\
\hline División 5-Salud & $28,9 \%$ & $27,8 \%$ & $29,0 \%$ & $28,1 \%$ \\
\hline División 6-Transporte y Comunicaciones & $29,8 \%$ & $20,6 \%$ y $34,1 \%$ & $22,9 \%$ у $35,3 \%$ & $27,1 \%$ y $28,7 \%$ \\
\hline División 7-Esparcimiento & $19,3 \%$ & $22,8 \%$ y $22,1 \%$ & $23,6 \%$ y $22,9 \%$ & $24,5 \%$ y $21,7 \%$ \\
\hline División 8-Enseñanza & $29,4 \%$ & $31,5 \%$ & $31,9 \%$ & $29,2 \%$ \\
\hline División 9-Bienes y Servicios Varios & $20,2 \%$ & $19,8 \%$ & $20,2 \%$ & $20,9 \%$ \\
\hline
\end{tabular}

Fuente: IPC-LP, FCEyJ, UNLPam

El cuadro incluye además la apertura para las 9 divisiones. Debe notarse aquí algo a lo que ya se hizo referencia en la introducción y es que el INDEC se adaptó en el transcurso de 2017 a las recomendaciones internacionales de tomar 12 divisiones en lugar de 9.

Tal es así que INDEC abre hoy la división "Alimentos y bebidas" en "Alimentos y bebidas no alcohólicas" por un lado, y "Bebidas alcohólicas y tabaco" por el otro; también abre "Transporte y comunicaciones" en "Transporte" por un lado y "Comunicaciones" por el otro; y abre "Esparcimiento" en "Recreación y cultura" por un lado y "Restaurantes y hoteles" por otro. Es por ello que el cuadro No. 6 presenta en tres celdas de las columnas de INDEC dos valores en lugar de uno. Si bien no nos permite esto hacer una comparación cuantitativa directa, sí podemos sacar algunas conclusiones.

Hecha la aclaración, podemos ahora analizar los datos. Comenzando por el IPC-LP, de estos incrementos acumulados en 12 meses, puede observarse que la división 1 "Alimentos y bebidas" creció por debajo, un 18,7 \%; mientras que la división 3 "Propiedades, combustibles, agua y electricidad" presentó el mayor incremento en 41,4\%. La división 2 "Indumentaria y calzados" es la división que menos ajustó sus precios con apenas 9,8 \% en estos 12 meses. 
El patrón en INDEC es similar, aunque las subas en las divisiones 2 y 3 fueron mayores que en el IPC-LP. "Propiedades, Combustibles, Agua y Electricidad" aumentó en estos 12 meses un 55,6 \% en INDEC Nacional, 56,4 \% en la región Pampeana y 48,8 \% en la región Patagónica.

Cabe señalar aquí que el gobierno nacional adoptó como política la quita de subsidios en servicios públicos al tiempo que incrementa las tarifas para los consumidores corrigiendo el atraso tarifario al que se hizo referencias más arriba. También es importante notar que este desequilibrio tarifario es mayor en Buenos Aires que en el interior del país, y por ello, es de esperarse que la estructura de precios en la división 3 reciba mayores incrementos en Buenos Aires que en las otras regiones. Dado que la región Pampeana incluye una porción de Buenos Aires, es posible que La Pampa reciba un peso de incrementos mayores a los que realmente existen en la Provincia.

Nuestro proyecto de investigación, de alguna manera justifica que el IPC-LP se construya durante estos próximos años de desinflación y ajustes tarifarios, porque de otro modo el sesgo de Buenos Aires le imprime a la Provincia de La Pampa incrementos de precios que en realidad no ocurren.

Una comparación del IPC-LP con estos dos IPC regionales de INDEC nos permite observar mayor compatibilidad con el IPC de la región Patagónica que aquella que existe con la región Pampeana, lo cual tiene sus fundamentos en el sesgo comentado. En la medida que el gobierno Nacional insista en corregir el histórico atraso tarifario de Buenos Aires frente al existente en el interior del país, los incrementos de precios serán mayores en esta región pampeana respecto de lo que sería realmente en La Pampa, más equilibrada en materia tarifaria que su provincia vecina.

Por otro lado, es sabido en Argentina que La Pampa se disputa en diversas cuestiones si pertenece realmente a esta región pampeana o patagónica, jugando un rol fundamental el proceso de integración regional declarado en la cumbre de Gobernadores Patagónicos, realizada en Santa Rosa, el 26 de Junio de 1996.

En cualquier caso, no corresponde aquí profundizar en esta divergencia, pero sí insistir que ninguna de las regiones que mide INDEC se ajustan a la realidad de La Pampa. Se podrá argumentar que lo mismo ocurre con otras provincias, porque en la medida que trabajamos con regiones, ninguna representa fielmente la realidad de una Provincia individual y concreta, pero fundamentamos aquí que hay una distorsión extraordinaria en esta región Pampeana que justifica se elabore el Índice de Precios al Consumidor local, hasta tanto se resuelva el desequilibrio tarifario de Buenos Aires, o al menos se equilibre con respecto al interior del país.

Un análisis más detallado podría identificar los artículos locales que presentan ajustes de precios diferentes al resto del país, pero estos son factores comunes a otras provincias, lo que nos parece de menor importancia que el factor señalado. 


\section{Conclusión}

En este trabajo analizamos la conveniencia de desarrollar un índice de precios local, provincial, tomando en cuenta la mayor transparencia que alcanzó el INDEC a partir de 2016.

Confirmamos la hipótesis de que se justifica elaborar un índice para La Pampa dado que su realidad no se encuentra representada en el índice Nacional, y tampoco en los dos regionales Pampeano y Patagónico que se elaboran desde mediados de 2017. El fundamento para esta conclusión se encuentra en que el IPC de la región Pampeana tiene un fuerte sesgo bonaerense en un período histórico donde el gobierno nacional intenta corregir las bajas tarifas de Buenos Aires frente a las existentes en el interior del país.

Nuevas correcciones en tarifas en Buenos Aires implican una mayor variación en los índices de precios Nacional y Región Pampeana de la que se presenta en la Provincia de La Pampa.

Mostramos que el IPC de la Región Patagónica resulta más representativo de La Pampa que el IPC de la Región Pampeana.

\section{Referencias bibliográficas}

Abraham, K. y Otros (2000). Price index research in the coming decades. en Monthly Labor Review, Vol. 123, Washington.

Armeknecht p. y Maitland - smith f. (2004). Producer Price Index Manual. Theory and Practice. ILO/OIT, Ginebra.

Camelo, H. (2001). Ingresos y gastos de consumo de los hogares en el marco del SCN y en encuestas a hogares. División de estadísticas y proyecciones económicas. CEPAL.

Comari, C. y Moyano, A. (2013). El Sistema Integrado de Encuestas a Hogares de Argentina. Claves para el sostenimiento de un sistema de estadísticas sociales abarcativo, robusto, ágil y coherente. XLI Coloquio Argentino de Estadística. Sociedad Argentina de Estadística (SAE)/Universidad Nacional de Cuyo (UNCu). Mendoza, Argentina.

.CEPAL (1986) "Encuestas de ingresos y gastos. Conceptos y métodos en la experiencia latinoamericana". Cuadernos de la CEPAL N 53.

Comisión Asesora para el Estudio del Índice de Precios al Consumidor (1995). Toward a More Accurate Measure of the Cost of Living. Informe Provisional al Comité de Finanzas del Senado, 15 de septiembre (Washington)

CCE-Eurostat, FMI, OCDE, Naciones Unidas, Banco Mundial (1993). Sistema de Cuentas Nacionales 1993. Bruselas/Luxemburgo, Nueva York, París, Washington DC.

Departamento Administrativo Nacional de Estadística (DANE) (1999), Metodología IPC 1998. Colombia.

Dirección General de Estadística y Censos (DGEyC) de la Ciudad de Buenos Aires (2009). Canastas de Consumo de la Ciudad de Buenos Aires. Metodología y cálculos iniciales.

Eurostat (2001), Compendium of HICP Reference Documents, Working Documents cat. N ${ }^{\circ}$ KSAO-01-005-EN-I, European Communities. 
FMI (2006), Manual de Índice de precios al consumidor, Teoría y Práctica.

FMl y otros (2009), Guía Práctica para el Establecimiento de índices de precios al consumidor, Washington D. C.

INDEC (1992). Encuesta Nacional urbana de Ingresos y Gastos de los Hogares. Conceptos y definiciones básicas para una nueva encuesta de ingresos y gastos.

INDEC (1998). Encuesta Nacional de Gastos de los Hogares. Total del país, regiones.

INDEC (1988). Encuesta de Gastos e Ingresos de los Hogares. Serie Estudios No 11.

INDEC (2001). Índice de Precios al Consumidor Gran Buenos Aires base 1999=100. Serie Metodología $N^{\circ} 13$, Instituto Nacional de Estadística y Censos.

INDEC (2005). Índice de Precios al Consumidor Nacional. Primera etapa, base 2003=100. Información de prensa, Instituto Nacional de Estadística y Censos.

INDEC (2007). Encuesta Nacional de Gastos de los Hogares 2004-2005. Base de datos de gastos de consumo e ingresos. Resumen metodológico. Instituto Nacional de Estadística y Censos.

INDEC (2011). Encuesta Nacional de Gasto a los Hogares en 2004/2005 y 2011. Análisis de los hábitos de consumo de la población de referencia de acuerdo a ambos relevamientos. Similitudes y diferencias. Evaluación factibilidad de utilización de la Encuesta 2011."

INDEC (2013). Encuesta Nacional de Gastos de los Hogares, ENGHo 2012/2013. Buenos Aires, 2013.

INDEC (2014a). Encuesta Nacional de Gastos de los Hogares. ENGHo 2012/2013.

INDEC (2014b). El Gasto de Consumo de los Hogares Urbanos en la Argentina. Un análisis a partir de las mediciones de 1996/1997, 2004/2005 y 2012/2013. Serie estudios No. 47.

INDEC (2014c). Índice de precios al consumidor Nacional Urbano (IPCNu). Serie documentos de trabajo No. 22.

INDEC, (2016a). Índice de Precios al Consumidor (IPC). Buenos Aires, abril de 2016. Disponible aquí:

https://www.indec.gob.ar/ftp/cuadros/economia/ipc_metodologia_abril2016.pdf

INDEC (2016b). Índice de Precios al Consumidor Gran Buenos Aires. Metodología INDEC No. 19, Buenos Aires, agosto 2016. Disponible aquí:

https://www.indec.gob.ar/ftp/cuadros/economia/ipc_metodologia19_agosto2016.pdf Instituto Brasileiro de Geografía e Estadistica (IBGE) (2007). Sistema Nacional de Índices de Preços ao Consumidor, Métodos de Cálculo - 5a edição. Série Relatórios Metodológicos, volume 14.

Instituto Nacional de Estadística y Censos, Costa Rica (INEC) (2006). Metodología del Índice de Precios al Consumidor, base julio 2006. San José, Costa Rica, septiembre.

Instituto Nacional de Estadísticas (2002), Metodología IPC 2001, Madrid, España.

Instituto Nacional de Estadísticas (2008), Metodología. VI Encuesta de Presupuestos Familiares 2006-2007, Santiago, Chile.

Instituto Nacional de Estadísticas (INE) Chile (1999). Estadística y Economía. Número especial Nuevo IPC, primer semestre.

Instituto Nacional de Estadísticas y Censos (INEC) (2005). Metodología del Índice de Precios al Consumidor IPC. Quito, Ecuador.

NACIONES UNIDAS (2001). Clasificaciones de gastos por finalidades: Clasificación del consumo 
individual por finalidades (CCIF). Serie M informes estadísticos N 84. División de Estadística, Nueva York.

NACIONES UNIDAS (2009). Guía práctica para el establecimiento de Índices de Precios al Consumidor. División de Estadística, Nueva York.

OECD (2002), Main Economic Indicators. Comparative Methodological Analysis: Consumer and Producer Price Indices, volume 2002, supplement 2, july.

Office for National Statistics (ONS) (2007). Consumer Price Indices Technical Manual. 2007 Edition, may, London.

OIT (2003), Informe III. "Índices de precios al consumidor", Decimoséptima Conferencia Internacional de Estadísticos del Trabajo, Ginebra 24 de noviembre a 3 de diciembre de 2003, ICLD/17/2003/3, Ginebra.

OIT/FMI/OCDE/Oficina Estadística de las Comunidades Europeas/Organización de las Naciones Unidas/Banco Mundial (2006), Manual del índice de precios al consumidor. Teoría y práctica, División de Español, Departamento de Tecnología y Servicios Generales, Washington, Fondo Monetario Internacional.

Pilorget, J. P. y otros (2013). Ponderaciones Híbridas: Propósitos, Ventajas y Desventajas de su Utilización en Índices de Precios. XLI Coloquio Argentino de Estadística. Sociedad Argentina de Estadística (SAE) / Universidad Nacional de Cuyo (UNCu). Mendoza, Argentina.

Statistics Canadá, Consumer Price Index, información disponible en su sitio web: http://www.statcan.gc.ca/start-debut-eng.html 